normal cognitive performance and MCI or dementia, with the latter being considered an absorbing state

Results During the study period, a quarter of participants progressed to MCI from the normal state. Being in the lowest quintile of wealth was associated with a lower probability of transitioning back to a normal cognitive state from MCI, compared with those in the highest quintile. Greater wealth was weakly associated with a lower risk of transitioning from normal cognitive state to MCI and from MCI to dementia.

Conclusion The overall results imply that socioeconomic advantage might be protective against rapid progression from mild to more severe neurocognitive disorders such as dementia in later life.This research indicates that older adults from different socioeconomic backgrounds have different probabilities of transitioning between different stages of neurocognitive disorders in a population sample and this could help us to define prevention strategies to delay cognitive impairment.

\section{P07 DOES SOCIAL MEDIA INFLUENCE ADOLESCENT ENGAGEMENT IN HEALTH RISK BEHAVIOURS? FINDINGS FROM A SYSTEMATIC REVIEW}

\footnotetext{
${ }^{1}$ Amrit Kaur Purba*, 'Paul M Henery, ${ }^{1}$ Rachel M Thomson, ${ }^{1}$ Anna Pearce, 1,2Marion Henderson, 'S Vittal Katikireddi. ${ }^{1}$ MRC/CSO Social and Public Health Sciences Unit, University of Glasgow, Glasgow, UK; ${ }^{2}$ School of Social Work and Social Policy, University of Strathclyde, Glasgow, UK
}

\subsection{6/jech-2021-SSMabstracts.97}

Background Social media (SM) may influence adolescents' perceived social norms and subsequent health risk behaviours, although the evidence base around this is still developing. We conducted a systematic review of the relationship between SM and adolescent health risk behaviours: alcohol/tobacco/drug use, e-cigarettes, diet, physical activity, antisocial behaviours, gambling, sexual risk behaviours and multiple health risk behaviours, in adolescents aged 10-19 years.

Methods We searched CINAHL, EMBASE, MEDLINE, APA PsycINFO, SocINDEX, preprint repositories and Google Scholar for studies published post-1996 reporting at least one relevant outcome with an SM measure (PROSPERO: CRD42020179766). Exposures of interest were time on SM, frequency of use, and exposure to health risk behaviour content (HRBC). Screening and risk of bias (RoB) were completed independently by two reviewers using a modified Newcastle Ottawa Scale. Following Cochrane guidance, we conducted synthesis based on direction of effects (benefit vs harm), sign testing and estimation of the proportion of datapoints reporting adverse effects (presented). Meta-analyses will produce average effect sizes (underway).

Results Of 13,150 hits, 84 studies were included. Twenty studies were low RoB, 27 moderate, and 38 high. Between studies all outcomes were addressed, the most common being alcohol use $(n=25)$ and sexual risk behaviours $(n=20)$. Twelve studies investigated $>1$ outcome. For alcohol use, most datapoints reported harmful effects of time spent (88.9\%; 95\% CI 56.5-98.0\%, p=0.04), frequency $(79.3 \%$; $61.6-90.2 \%, \mathrm{p}=0.002)$, exposure to HRBC $(100.0 \%$; $75.8-$ $100.0 \%, \mathrm{p}<0.001)$ and other SM activity measures $(81.8 \%$; 52.3-94.9\%, $\mathrm{p}=0.07)$. Datapoints examining sexual risk behaviours mostly reported harmful effects of time $(75.0 \%$; $30.1-95.4 \%, \quad \mathrm{p}=0.63)$, frequency $(91.7 \%$; 64.6-98.5\%, $\mathrm{p}=0.006), \quad$ HRBC $(100.0 \% ; 43.9-100.0 \%, p=0.25)$, and other SM activity $(76.2 \% ; 61.5-86.5 \%, \mathrm{p}<0.001)$. For e-cigarettes $(n=8)$ and antisocial behaviour $(n=17)$, all datapoints reported harmful effects of SM (e-cigarettes 95\% CI 67.6$100.0 \%, \quad \mathrm{p}=0.008$; antisocial behaviour 95\% CI $81.6-$ $100.0 \%, \mathrm{p}<0.001)$. Across all outcomes, exposure to HRBC on SM was most likely to report a harmful effect $(100.0$ vs $83.0 \%$ for other exposures, $\mathrm{p}=0.0062)$. Harmful effects were similar for datapoints at high $(85.6 \%)$ and low/moderate (86.7\%) RoB.

Conclusion SM use is adversely associated with adolescent health risk behaviours, particularly exposure to content pertaining to these behaviours. The current evidence base is limited by methodological weaknesses, including a lack of longitudinal data (risking reverse causation) and future robust research to assess causality is needed. Given the increasing targeting of SM by unhealthy commodity industries, available evidence suggests action to reduce the risk adolescents face from exposure to health risk behaviours is needed.

\section{P08 SOCIAL MEDIA USE AND SOCIAL CONNECTEDNESS IN ADOLESCENCE: RISKS AND BENEFITS}

${ }^{1}$ Lizzy Winstone*, ${ }^{1,2}$ Becky Mars, ${ }^{2,3}$ Claire MA Haworth, ${ }^{1} J u d i$ Kidger. ${ }^{1}$ Population Health Science, University of Bristol, Bristol, UK; ${ }^{2}$ NIHR Biomedical Research Centre, University Hospitals Bristol NHS Foundation Trust, Bristol, UK; ${ }^{3}$ Psychological Science, University of Bristol, Bristol, UK

\subsection{6/jech-2021-SSMabstracts.98}

Background Connectedness to school, family and peers is a key determinant of adolescent mental health. The relationship between social media use (SMU) and social connectedness is complex, potentially improving closeness to peers, whilst possibly diminishing school connectedness. Evidence to date has been piecemeal and contradictory with particular gaps in research on school and family connectedness. In this qualitative study we explore the relationship between SMU and these three areas of social connectedness using the Displacement Hypothesis and the Stimulation Hypothesis as competing theoretical lenses.

Methods In-depth paired and individual interviews were conducted with nineteen girls and five boys aged 13-14 years in two English secondary schools. Interviews covered various topics relating to SMU and well-being. Interviews were transcribed verbatim, coded and thematically analysed.

Results Thematic analysis of the transcripts identified six themes: (i) 'Time displacement', (ii) '(Mis)Trust', (iii) 'Generational disconnect', (iv) 'Personal and group identity', (v) 'Keeping in touch', and (vi) 'Social obligation'. Results indicated support for both the Displacement and Stimulation Hypotheses. School connectedness was undermined through displacement of time spent on homework and feeling misunderstood by teachers, but enhanced by maintaining relationships with classmates. Family connectedness appeared to be weakened through the same feeling of being misunderstood by parents, not feeling trusted to responsibly navigate SMU or displacing time spent together. However, SMU also provided opportunities for parents to demonstrate trust, to share in entertainment and allowed young people to stay in contact with family members overseas. In line with the Stimulation Hypothesis, connectedness to close friends was strengthened through selfdisclosure and a sense of shared identity, but broader peer relationships were undermined by feelings of mistrust. 